\title{
RECURSIVE FORMULAE FOR SOME BIVARIATE COUNTING DISTRIBUTIONS OBTAINED BY THE TRIVARIATE REDUCTION METHOD
}

\author{
BY \\ J.F. WALHIN** AND J. PARIS* \\ * Université Catholique de Louvain \\ - Secura Belgian Re
}

\begin{abstract}
In this paper we study some bivariate counting distributions that are obtained by the trivariate reduction method. We work with Poisson compound distributions and we use their good properties in order to derive recursive algorithms for the bivariate distribution and bivariate aggregate claims distribution. A data set is also fitted.
\end{abstract}

\section{KEYWORDS}

Bivariate counting distributions, Poisson compound distribution, mixed Poisson distribution, Hofmann distribution, recursive algorithms, fit.

\section{INTRODUCTION}

Ahmed (1961) and Papageorgiou and David (1995) discuss some bivariate counting distributions, namely, the joint distribution $(N, M)$ where $N=N_{0}+N_{1}$ and $M=N_{0}+N_{2}$ with $N_{0}, N_{1}$ and $N_{2}$ independent random variables such that

$$
\begin{aligned}
& \mathbb{P}\left(N_{0}=n\right)=\int_{0}^{\infty} \frac{e^{-\lambda}}{n !} \frac{\lambda^{n}}{n !} d U(\lambda), \quad n \geq 0 \\
& \mathbb{P}\left(N_{1}=n\right)=e^{-\lambda_{1}} \frac{\lambda_{1}^{n}}{n !}, \quad n \geq 0 \\
& \mathbb{P}\left(N_{2}=n\right)=e^{-\lambda_{2}} \frac{\lambda_{2}^{n}}{n !}, \quad n \geq 0
\end{aligned}
$$

i.e. $N_{1}$ and $N_{2}$ are Poisson distributed while $N_{0}$ is mixed Poisson distributed with mixing distribution whose cumulative density function (cdf) is $U(\lambda)$. 
The joint probability function (pf) of $(N, M)$ is given by

$$
\mathbb{P}(N=n, M=m)=\sum_{k=0}^{\min (n, m)} \mathbb{P}\left(N_{0}=k\right) \mathbb{P}\left(N_{1}=n-k\right) \mathbb{P}\left(N_{2}=m-k\right)
$$

For some choices of the mixing distribution $\Lambda$, Papageorgiou and David (1995) give the density of $(N, M)$ by using Stirling numbers of the second kind, C-numbers and modified Bessel functions of the third kind.

Using a general class of counting random variables that are simultaneously mixed and compound Poisson, it is possible to give simple expressions for the joint distribution of $(N, M)$ which avoid these numbers. Moreover our methodology gives easily the joint pf of the random sums

$$
\left(S_{N}, S_{M}\right)=\left(X_{1}+\ldots+X_{N}, Y_{1}+\ldots+Y_{M}\right)
$$

where $X_{1}, X_{2}, \ldots$ (resp. $Y_{1}, Y_{2}, \ldots$ ) is a random sample of observations from $X$ (resp. $Y$ ).

$X$ and $Y$ are independent nonnegative arithmetic random variables that are also independent of $(N, M)$. The distribution of $\left(S_{N}, S_{M}\right)$ is of interest in insurance problems where it represents the aggregate claims distributions when $X$ and $Y$ are claim amounts.

We will also extend the model to $(N, M)=\left(N_{0}+N_{1}, N_{0}+N_{2}\right)$ where $N_{0}, N_{1}$ and $N_{2}$ are mixed Poisson distributions.

Finally a data set will be fitted.
We will use the following conventions: $\sum_{k=a}^{b}=0$ when $b<a$ and
$N=n, M=m)=0$ when $n<0$ or $m<0$. $\mathbb{P}(N=n, M=m)=0$ when $n<0$ or $m<0$.

In order to prove the algorithms leading to recursive formulae for some compound distributions, we will use extensively the concept of ordinary generating function (see Panjer and Willmot (1992) for a reference in actuarial sciences).

Let a sequence $\left\{a_{n}, n=0,1,2, \ldots\right\}$ of real numbers.

The ordinary generating function of this sequence is defined as

$$
T_{a_{n}}(z)=\sum_{n=0}^{\infty} a_{n} z^{n}
$$

Of course $z$ must be chosen such that the sum exists.

Ordinary generating functions have the following nice properties:

- There is a one-to-one correspondence between $\left\{a_{n}, n=0,1,2, \ldots\right\}$ and $T_{a_{n}}(z)$

- $a_{n}=\left.\frac{1}{n !} \frac{d^{n} T_{a_{n}}(z)}{d z^{n}}\right|_{z=0}$

- $c_{n}=\alpha a_{n}+\beta b_{n} \Leftrightarrow T_{c_{n}}(z)=\alpha T_{a_{n}}(z)+\beta T_{b_{n}}(z)$

- $c_{n}=\sum_{k=0}^{n} a_{k} b_{n-k} \Leftrightarrow T_{c_{n}}(z)=T_{a_{n}}(z) T_{b_{n}}(z)$

- $T_{n a_{n}}(z)=z \frac{d}{d z} T_{a_{n}}(z)$ 
The philosophy for using ordinary generating functions is the following:

- we look for a relation between some sequences $a_{n}, b_{n}, c_{n}, \ldots$

- go in the $z$ map where the calculations become easier (think of the convolution that becomes a product)

- go back to the initial map by inverting the expression in $z$ thanks to the properties.

The notion of ordinary generating function and its properties trivially extend in a bivariate setting.

In this paper, the sequence $a_{n}$ or $a_{(n, m)}$ in a bivariate setting will be probability functions. As a consequence we will not have problems of convergence for the ordinary generating functions: $|z|<\infty$.

In the present case, ordinary generating functions are just probability generating functions (pgf).

From now on we will only refer to pgf and we will use them extensively in the sense of ordinary generating functions.

\section{A GENERAL FAMILY OF RANDOM VARIABLES THAT ARE SIMULTANEOUSLY MIXED AND COMPOUND POISSON}

Walhin and Paris (2000b) review the characteristics of a general family of random variables that have the property of being mixed and compound Poisson distributions.

A mixed Poisson process is such that

$$
\Pi(n, t)=\mathbb{P}(N(t)=n)=\int_{0}^{\infty} e^{-\lambda t} \frac{(\lambda t)^{n}}{n !} d U(\lambda)
$$

where $N(t)$ gives the number of occurrences in $(0, t]$.

By choosing

$$
\begin{aligned}
& \Pi(0, t)=e^{-\theta(t)} \\
& \theta(t) \geq 0 \\
& \theta(0)=0 \\
& \frac{d}{d t} \theta(t) \text { completely monotone }
\end{aligned}
$$

Walhin and Paris (2000b) show that $N(t)$ can also be interpreted as a compound Poisson model:

$$
N(t)=\sum_{i=1}^{L(t)} \xi_{i}
$$


where $L(t)$ is Poisson distributed and independent of the $\xi_{i}$ which are independent identically distributed (i.i.d.) random variables. We will use this property in the sequel. A good choice for the function $\theta^{\prime}(t)$ is the choice made by Hofmann (1955) and studied in Walhin and Paris (2000b) and in Kestemont and Paris (1985):

$$
\theta^{\prime}(t)=\frac{p}{(1+c t)^{a}} \quad p>0, \quad c>0, \quad a \geq 0
$$

By integration, one has

$$
\begin{aligned}
& \theta(t)=\frac{p}{c(1-a)}\left[(1+c t)^{1-a}-1\right] \\
& \theta(t)=\frac{p}{c} \ln (1+c t) \quad \text { by continuity for } a=1
\end{aligned}
$$

Particular cases of interest are Poisson $(a=0)$, Poisson Inverse Gaussian $(a=0.5)$, Negative Binomial $(a=1)$, Polya-Aeppli $(a=2)$ and Neymann Type $\mathrm{A}(a \rightarrow \infty, c \rightarrow 0, a c \rightarrow b)$.

Some properties are

$$
\begin{aligned}
\psi_{N(t)}(z) & =\sum_{n=0}^{\infty} \Pi(n, t) z^{n}=\Pi(0, t-t z)=e^{-\theta(t-t z)} \\
E N(t) & =p t \\
\operatorname{Var} N(t) & =p t+p a c t^{2} \\
\psi_{N(t)}(z) & =e^{-\theta(t)\left(1-\psi_{\xi}(z)\right)}
\end{aligned}
$$

where $\psi_{X}(z)=\mathbb{E}\left[z^{X}\right]$ denotes the pgf of the random variable $X$. The probability law of the $\xi_{i}$ is deduced from

$$
\begin{aligned}
q(0) & =0 \\
\psi_{\xi}(z) & =\sum_{k=1}^{\infty} q(k) z^{k}=1-\frac{\theta(t-t z)}{\theta(t)} \\
\frac{q(n)}{q(n-1)} & =r+\frac{s}{n}, \quad n>1 \\
r & =\frac{c t}{1+c t} \\
s & =r(a-2)
\end{aligned}
$$

One says that the $\xi_{i}$ belong to the $(r, s, 1)$ class. The 1 in $(r, s, 1)$ is connected with the $n>1$ in (3). 
From now on we will refer to the Hofmann distribution $(H o(p, c, a))$ with the convention that $t=1$. In model $1, N_{0}$ will be $H o(p, c, a)$ while in model $2, N_{i}$ will be $H o\left(p_{i}, c_{i}, a_{i}\right), i=0,1,2$. Note that in general, the $(r, s, 1)$ class is denoted as $(a, b, 1)$ class. We use the notation $(r, s, 1)$ class in order to avoid confusion with the $a$ of the Hofmann distribution.

\section{MODEL 1: BIVARIATE COUNTING VARIABLES}

In this section we work with the model

$$
(N, M)=\left(N_{0}+N_{1}, N_{0}+N_{2}\right)
$$

where $N_{0}$ is $H o(p, c, a)$ and $N_{1}$ and $N_{2}$ are respectively $\operatorname{Po}\left(\lambda_{1}\right)$ and $\operatorname{Po}\left(\lambda_{2}\right)$. The three random variables are assumed to be mutually independent.

Let

$$
\phi(u, v)=\sum_{n=0}^{\infty} \sum_{m=0}^{\infty} p(n, m) u^{n} v^{m}
$$

be the pgf of $(N, M)$ where we use the notation

$$
p(n, m)=\mathbb{P}[N=n, M=m]
$$

Let $\psi_{0}, \psi_{1}$ and $\psi_{2}$ the pgf of $N_{0}, N_{1}$ and $N_{2}$ respectively.

We have

$$
\begin{aligned}
\phi(u, v) & =\mathbb{E}\left[u^{N} v^{M}\right]=\mathbb{E}\left[(u v)^{N_{0}} u^{N_{1}} v^{N_{2}}\right] \\
& =\psi_{0}(u v) \psi_{1}(u) \psi_{2}(v) \\
& =e^{-\theta(1)\left[1-\psi_{\xi}(u v)\right]} e^{-\lambda_{1}(1-u)} e^{-\lambda_{2}(1-v)}
\end{aligned}
$$

Differentiating with respect to $u$ and multiplying by $u$ gives

$$
u \frac{\partial \phi(u, v)}{\partial u}=\theta(1) u v \frac{\partial \psi_{\xi}(u v)}{\partial u} \phi(u, v)+u \lambda_{1} \phi(u, v)
$$

Inverting this expression gives

$$
n p(n, m)=\theta(1) \sum_{k=1}^{\min (n, m)} k q(k) p(n-k, m-k)+\lambda_{1} p(n-1, m), \quad n>0
$$

Differentiating with respect to $v$ gives a symmetric recursion.

We have proved 


\section{Theorem 1}

For model 1 the probability function is given by the following recursion

$$
\begin{aligned}
p(0,0) & =e^{-\theta(1)-\lambda_{1}-\lambda_{2}} \\
n p(n, m) & =\theta(1) \sum_{k=1}^{\min (n, m)} k q(k) p(n-k, m-k)+\lambda_{1} p(n-1, m), \quad n>0 \\
m p(n, m) & =\theta(1) \sum_{k=1}^{\min (n, m)} k q(k) p(n-k, m-k)+\lambda_{2} p(n, m-1), \quad m>0
\end{aligned}
$$

Let us note that for the particular cases where $N_{0}$ is Negative Binomial or Poisson Inverse Gaussian, we have easier recursions.

The case Negative Binomial $(a=1)$ is given in Hesselager (1996) where the fact that the Negative Binomial belongs to the $(r, s, 0)$ class is used:

$$
\begin{gathered}
\mathbb{P}\left(N_{0}=0\right)=(1+c)^{-\frac{p}{c}} \\
\frac{\mathbb{P}\left(N_{0}=k\right)}{\mathbb{P}\left(N_{0}=k-1\right)}=r+\frac{s}{k}, \quad k>0
\end{gathered}
$$

with

$$
\begin{aligned}
& r=\frac{c}{1+c} \\
& s=\frac{p-c}{1+c}
\end{aligned}
$$

The Negative Binomial can be expressed in the following explicit notation:

$$
\mathbb{P}\left(N_{0}=k\right)=\frac{(p / c)(p / c+1) \ldots(p / c+k-1)}{k !}\left(\frac{c}{1+c}\right)^{k}\left(\frac{1}{1+c}\right)^{(p / c)}
$$

In this case the recursion becomes (Hesselager (1996)):

$$
\begin{aligned}
p(0,0)= & (1+c)^{-\frac{p}{c}} e^{-\lambda_{1}-\lambda_{2}} \\
p(n, m)= & \left(r+\frac{s}{n}\right) p(n-1, m-1)+\frac{\lambda_{1}}{n} p(n-1, m) \\
& -\frac{\lambda_{1} r}{n} p(n-2, m-1), \quad n>0 \\
p(n, m)= & \left(r+\frac{s}{m}\right) p(n-1, m-1)+\frac{\lambda_{2}}{m} p(n, m-1) \\
& -\frac{\lambda_{2} r}{m} p(n-1, m-2), \quad m>0
\end{aligned}
$$


The case Poisson Inverse Gaussian $(a=0.5)$ is derived without using the distribution of the $\xi_{i}$ (see equations (1) and (2)). The pgf of $(N, M)$ has the following direct properties:

$$
\begin{aligned}
\phi(u, v)= & e^{-\frac{2 p}{c}\left((1+c(1-u v))^{\frac{1}{2}}-1\right)} e^{-\lambda_{1}(1-u)} e^{-\lambda_{2}(1-v)} \\
\frac{\partial}{\partial u} \phi(u, v)= & \left(\lambda_{1}+\frac{p v}{(1+c(1-u v))^{\frac{1}{2}}}\right) \phi(u, v) \\
\frac{\partial^{2}}{\partial u^{2}} \phi(u, v)= & \lambda_{1}^{2} \phi(u, v)+\frac{1}{2} \frac{c p v^{2}}{(1+c(1-u v))^{\frac{3}{2}}} \phi(u, v) \\
& +\frac{p^{2} v^{2}}{1+c(1-u v)} \phi(u, v)+\frac{2 \lambda_{1} p v}{(1+c(1-u v))^{\frac{1}{2}}} \phi(u, v) \\
\text { (5) }(1+c(1-u v)) \frac{\partial^{2}}{\partial u^{2}} \phi(u, v)= & \phi(u, v)\left(-\lambda_{1}^{2}(1+c)+\lambda_{1}^{2} c u v-\frac{1}{2} \lambda_{1} c v+p^{2} v^{2}\right) \\
& +\frac{\partial}{\partial u} \phi(u, v)\left(2 \lambda_{1}(1+c)-2 \lambda_{1} c u v+\frac{1}{2} c v\right)
\end{aligned}
$$

From (4) we easily find the initializing terms:

$$
\begin{aligned}
& p(0,0)=e^{-\lambda_{1}-\lambda_{2}-2 \frac{p}{c}\left((1+c)^{0.5}-1\right)} \\
& p(1,0)=\lambda_{1} p(0,0) \\
& p(0,1)=\lambda_{2} p(0,0) \\
& p(1,1)=\left(\lambda_{1} \lambda_{2}+\frac{p}{(1+c)^{\frac{1}{2}}}\right) p(0,0)
\end{aligned}
$$

Inverting (5) and using its similar expression in $v$ gives

$$
\begin{aligned}
(1+c) n(n-1) p(n, m)= & c(n-1)\left(n-\frac{3}{2}\right) p(n-1, m-1)-\lambda_{1} c\left(2 n-\frac{7}{2}\right) p(n-2, m-1) \\
& +2 \lambda_{1}(1+c)(n-1) p(n-1, m)-\lambda_{1}^{2}(1+c) p(n-2, m) \\
& +\lambda_{1}^{2} c p(n-3, m-1)+p^{2} p(n-2, m-2), \quad n \geq 2 \\
(1+c) m(m-1) p(n, m)= & c(m-1)\left(m-\frac{3}{2}\right) p(n-1, m-1)-\lambda_{2} c\left(2 m-\frac{7}{2}\right) p(n-1, m-2) \\
& +2 \lambda_{2}(1+c)(m-1) p(n, m-1)-\lambda_{2}^{2}(1+c) p(n, m-2) \\
& +\lambda_{2}^{2} c p(n-1, m-3)+p^{2} p(n-2, m-2), \quad m \geq 2
\end{aligned}
$$

We recall that $p$ is one of the parameters of the Hofmann distribution $(H o(p, c, a))$ while $p(n, m)$ is the pf of $(N, M)$. 


\section{MODEL 1: BIVARIATE RANDOM SUMS}

Now let us study the bivariate vector

$$
\left(S_{N}, S_{M}\right)=\left(X_{1}+\ldots+X_{N_{0}+N_{1}}, Y_{1}+\ldots+Y_{N_{0}+N_{2}}\right)
$$

whose pf is given by

$$
\mathbb{P}\left[S_{N}=x, S_{M}=y\right]=g(x, y)=\sum_{n=0}^{\infty} \sum_{m=0}^{\infty} p(n, m) f_{X}^{* n}(x) f_{Y}^{* m}(y)
$$

where $f_{X}(x)$ (resp. $f_{Y}(y)$ ) is the pf of $X$ (resp. Y). Our aim is to give a recursive scheme in order to derive the $\mathrm{pf} g(x, y)$.

Let $\Phi(u, v)$ be the pgf of $\left(S_{N}, S_{M}\right)$ and let $\psi_{X}(u)$ (resp. $\left.\psi_{Y}(v)\right)$ be the pgf of $X$ (resp. Y).

We have

$$
\begin{aligned}
\Phi(u, v) & =\sum_{n=0}^{\infty} \sum_{m=0}^{\infty} p(n, m) \psi_{X}^{n}(u) \psi_{Y}^{m}(v) \\
& =\phi\left(\psi_{X}(u), \psi_{Y}(v)\right) \\
& =e^{-\theta(1)\left[1-\psi_{\xi}\left(\psi_{X}(u) \psi_{Y}(v)\right)\right]} e^{-\lambda_{1}\left(1-\psi_{X}(u)\right)} e^{-\lambda_{2}\left(1-\psi_{Y}(v)\right)}
\end{aligned}
$$

Differentiating with respect to $u$ and multiplying by $u$ gives

$$
u \frac{\partial \Phi(u, v)}{\partial u}=\theta(1) u \frac{\partial \Psi_{\xi}(u, v)}{\partial u} \Phi(u, v)+\lambda_{1} u \frac{\partial \psi_{X}(u)}{\partial u} \Phi(u, v)
$$

where $\Psi_{\xi}(u, v)=\psi_{\xi}\left(\psi_{X}(u) \psi_{Y}(v)\right)$ is the probability generating function of the pair

$$
\left(X_{1}+\ldots+X_{\xi}, Y_{1}+\ldots+Y_{\xi}\right)
$$

whose pf will be denoted by

$$
h(i, j)=\mathbb{P}\left(X_{1}+\ldots+X_{\xi}=i, Y_{1}+\ldots+Y_{\xi}=j\right), \quad i \geq 0, \quad j \geq 0
$$

Inverting (7) gives

(8) $x g(x, y)=\theta(1) \sum_{i=0}^{x} \sum_{j=0}^{y} i h(i, j) g(x-i, y-j)+\lambda_{1} \sum_{i=0}^{x} i f_{X}(i) g(x-i, y), \quad x>0$

The following theorem is a trivial extension of the bivariate Panjer (1981) algorithm given in Walhin and Paris (2000a). The proof is given for illustration. 


\section{Theorem 2}

The probability function $h(x, y)$ of

$$
\left(X_{1}+\ldots+X_{\xi}, Y_{1}+\ldots+Y_{\xi}\right)
$$

is given by

(9) $\quad h(0,0)=1-\frac{\theta\left(1-f_{X}(0) f_{Y}(0)\right)}{\theta(1)}$

(10) $h(x, y)=\frac{1}{1-r f_{X}(0) f_{Y}(0)}$

$$
\left(\sum_{i}^{x} \sum_{j}^{y}\left(r+s \frac{i}{x}\right) f_{X}(i) f_{Y}(j) h(x-i, y-j)+q(1) f_{X}(x) f_{Y}(y)\right), \quad x>0
$$

(11) $h(x, y)=\frac{1}{1-r f_{X}(0) f_{Y}(0)}$

$$
\left(\sum_{i}^{x} \sum_{j}^{y}\left(r+\frac{j}{y}\right) f_{X}(i) f_{Y}(j) h(x-i, y-j)+q(1) f_{X}(x) f_{Y}(y)\right), \quad y>0
$$

where we use the notation

$$
\sum_{i}^{x} \sum_{j}^{y} w(i, j)=\sum_{i=0}^{x} \sum_{j=0}^{y} w(i, j)-w(0,0)
$$

\section{Proof}

Equation (9) follows immediately from equation (2).

Now we prove equation (10). We have

$$
k q(k)=r(k-1) q(k-1)+(r+s) q(k-1), \quad k>1
$$

Multiplying each side of (12) by $\psi_{X}^{k-1}(u) \frac{d}{d u} \psi_{X}(u) \psi_{Y}^{k}(v)$ and summing from $k=1$ to $k=\infty$ we find

$$
\begin{aligned}
\frac{\partial}{\partial u} \Psi_{\xi}(u, v)-q(1) \frac{d}{d u} \psi_{X}(u) \psi_{Y}(v)= & r \frac{\partial}{\partial u} \Psi_{\xi}(u, v) \psi_{X}(u) \psi_{Y}(v) \\
& +(r+s) \Psi_{\xi}(u, v) \frac{d}{d u} \psi_{X}(u) \psi_{Y}(v) \\
& -(r+s) q(0) \frac{d}{d u} \psi_{X}(u) \psi_{Y}(v)
\end{aligned}
$$


Multiplying by $u$ and inverting gives

$$
\begin{aligned}
x h(x, y)= & r \sum_{i=0}^{x} \sum_{j=0}^{y}(x-i) f_{X}(i) f_{Y}(j) h(x-i, y-j) \\
& +(r+s) \sum_{i=0}^{x} \sum_{j=0}^{y} i f_{X}(i) f_{Y}(j) h(x-i, y-j) \\
& +q(1) x f_{X}(x) f_{Y}(y)
\end{aligned}
$$

Rearranging gives (10).

(11) follows similarly.

Of course more general results can be derived if (12) becomes

$$
k q(k)=r(k-1) q(k-1)+(r+s) q(k-1), \quad k>m
$$

for a general $m$. In this case, one says that the $\xi_{i}$ belong to the $(r, s, m)$ class.

With the symmetric expression of (8) we have the following result:

\section{Theorem 3}

For the model 1, the probability function $g(x, y)$ of the compound distribution is given by the following recursion:

$$
\begin{aligned}
g(0,0)= & e^{-\theta\left(1-f_{X}(0) f_{Y}(0)\right)} e^{-\lambda_{1}\left(1-f_{X}(0)\right)} e^{-\lambda_{2}\left(1-f_{Y}(0)\right)} \\
g(x, y)= & \theta(1) \sum_{i=1}^{x} \sum_{j=0}^{y} \frac{i}{x} h(i, j) g(x-i, y-j) \\
& +\lambda_{1} \sum_{i=1}^{x} \frac{i}{x} f_{X}(i) g(x-i, y), \quad x>0 \\
g(x, y)= & \theta(1) \sum_{i=0}^{x} \sum_{j=1}^{y} \frac{j}{y} h(i, j) g(x-i, y-j) \\
& +\lambda_{2} \sum_{j=1}^{y} \frac{j}{y} f_{Y}(j) g(x, y-j), \quad y>0
\end{aligned}
$$

where $h(i, j)$ is given by theorem 2 .

Proof

Equation (13) is immediately derived from equations (6) and (2).

Equation (14) follows immediately from equation (8) while its similar expression valid for $y>0$ gives (15). 


\section{MODEL 2}

In this section we consider the following model

$$
(N, M)=\left(N_{0}+N_{1}, N_{0}+N_{2}\right)
$$

where $N_{i}$ are independent $H o\left(p_{i}, c_{i}, a_{i}\right)$. The corresponding pf of the $\xi_{i}$ are denoted by $q_{i}$.

Then we have the following results. The proofs are similar to those given in sections 3 and 4 . So we omit them.

\section{Theorem 4}

For model 2, the probability function $p(n, m)$ is given by the following recursion:

$$
\begin{aligned}
& p(0,0)=e^{-\theta_{0}(1)-\theta_{1}(1)-\theta_{2}(1)} \\
& p(n, m)=\sum_{i=1}^{\min (n, m)} \frac{i}{n} \theta_{0}(1) q_{0}(i) p(n-i, m-i)+\sum_{i=1}^{n} \frac{i}{n} \theta_{1}(1) q_{1}(i) p(n-i, m), n>0 \\
& p(n, m)=\sum_{i=1}^{\min (n, m)} \frac{i}{m} \theta_{0}(1) q_{0}(i) p(n-i, m-i)+\sum_{i=1}^{m} \frac{i}{m} \theta_{2}(1) q_{2}(i) p(n, m-i), m>0
\end{aligned}
$$

\section{Theorem 5}

For model 2, the probability function $g(x, y)$ is given by the following recursion:

$$
\begin{aligned}
& g(0,0)=e^{-\theta_{0}\left(1-f_{X}(0) f_{Y}(0)\right)} e^{-\theta_{1}\left(1-f_{X}(0)\right)} e^{-\theta_{2}\left(1-f_{Y}(0)\right)} \\
& g(x, y)=\theta_{0}(1) \sum_{i=1}^{x} \sum_{j=0}^{y} \frac{i}{x} h(i, j) g(x-i, y-j)+\theta_{1}(1) \sum_{i=1}^{x} \frac{i}{x} b_{X}(i) g(x-i, y), \quad x>0 \\
& g(x, y)=\theta_{0}(1) \sum_{i=0}^{x} \sum_{j=1}^{y} \frac{j}{y} h(i, j) g(x-i, y-j)+\theta_{2}(1) \sum_{j=1}^{y} \frac{j}{y} b_{Y}(j) g(x, y-j), \quad y>0
\end{aligned}
$$

where $h(i, j)$ is given by theorem 2 and $b_{X}(k)$ is given by

$$
\begin{aligned}
& b_{X}(0)=1-\frac{\theta_{1}\left(1-f_{X}(0)\right)}{\theta_{1}(1)} \\
& b_{X}(k)=\frac{1}{1-r_{1} f_{X}(0)}\left(\sum_{i=1}^{k}\left(r+s \frac{i}{k}\right) f_{X}(i) b_{X}(k-i)+q_{1}(1) f_{X}(k)\right), \quad k>0
\end{aligned}
$$

and $b_{Y}(k)$ is defined similarly. 
Remark: for the case where the $N_{i}$ belong to the $(r, s, 0)$ class, Hesselager (1996) gives an easier algorithm. However, for the case Negative Binomial which is a member of the $(r, s, 0)$ class, numerical examples show that this algorithm is not stable while the combination of theorems 5 and 2 give stable recursions.

\section{A FIT}

We use in this section a set of accident data used in Papageorgiou and David (1995) for illustration.

We fit model 1 for the following choices of $N_{0}$ :

$-a=0$ : Poisson

- $a=1:$ Negative Binomial

- $a=0.5$ : Poisson Inverse Gaussian

- $a$ free: general Hofmann distribution.

The fits are proceeded by maximum likelihood. $N$ and $M$ are accident observations.

It can be shown that

$$
\begin{aligned}
& \hat{p}+\hat{\lambda}_{1}=\bar{N} \\
& \hat{p}+\hat{\lambda}_{2}=\bar{M}
\end{aligned}
$$

where $\bar{N}$ (resp. $\bar{M}$ ) is the empirical mean of $N$ (resp. $M$ ).

This reduces the number of estimates to be found by numerical techniques. For the Hofmann fit we need to maximize numerically the loglikelihood subject to three variables.

We find the following estimates:

TABLE 1

MaXimum LIKELIHOOd ADJUSTMENT

\begin{tabular}{lcccccc}
\hline \hline & $\lambda_{1}$ & $\lambda_{2}$ & $\boldsymbol{p}$ & $\boldsymbol{c}$ & \multicolumn{1}{c}{$\boldsymbol{a}$} & loglikelihood \\
\hline Poisson & 1.0319 & 1.2724 & 0.6388 & & 0 & -112.7380 \\
PIG & 1.0893 & 1.3298 & 0.5815 & 0.8432 & 0.5 & -112.3577 \\
NB & 1.0939 & 1.3344 & 0.5769 & 0.4092 & 1 & -112.3802 \\
Hofmann & 1.0796 & 1.3201 & 0.5912 & 1.6697 & 0.2546 & -112.3467 \\
\hline
\end{tabular}

Based on the comparison of the loglikelihoods, it does not seem necessary to work with a more complicated model than the one obtained with all $N_{i}$, $i=0,1,2$ being Poisson distributed. A likelihood ratio test would not reject this hypothesis.

The original data with fitted values are in the next table: 
TABLE 2

OBSERVED AND FITTED DISTRIBUTIONS FOR THE NUMBER OF ACCIDENTS

\begin{tabular}{|c|c|c|c|c|c|c|c|c|c|}
\hline$N$ & $\boldsymbol{M}$ & 0 & 1 & 2 & 3 & 4 & 5 & 6 & 7 \\
\hline \multirow[t]{5}{*}{0} & $o . b s$ & 5 & 6 & 4 & 1 & 1 & 0 & 0 & 0 \\
\hline & $a=0$ & 4.16 & 5.30 & 3.37 & 1.43 & 0.45 & 0.12 & 0.02 & 0.00 \\
\hline & $a=0.5$ & 4.29 & 5.71 & 3.80 & 1.68 & 0.56 & 0.15 & 0.03 & 0.01 \\
\hline & $a=1$ & 4.29 & 5.73 & 3.82 & 1.70 & 0.57 & 0.15 & 0.03 & 0.01 \\
\hline & $a=0.2546$ & 4.29 & 5.67 & 3.74 & 1.65 & 0.54 & 0.14 & 0.03 & 0.01 \\
\hline \multirow[t]{5}{*}{1} & $o b s$ & 4 & 9 & 3 & 4 & 3 & 0 & 0 & 0 \\
\hline & $a=0$ & 4.30 & 8.13 & 6.86 & 3.63 & 1.38 & 0.41 & 0.10 & 0.02 \\
\hline & $a=0.5$ & 4.68 & 8.06 & 6.58 & 3.46 & 1.33 & 0.40 & 0.10 & 0.02 \\
\hline & $a=1$ & 4.70 & 8.03 & 6.53 & 3.43 & 1.32 & 0.40 & 0.10 & 0.02 \\
\hline & $a=0.2546$ & 4.63 & 8.10 & 6.65 & 3.50 & 1.34 & 0.41 & 0.10 & 0.02 \\
\hline \multirow[t]{5}{*}{2} & $o b s$ & 2 & 5 & 5 & 4 & 2 & 0 & 0 & 0 \\
\hline & $a=0$ & 2.22 & 5.56 & 6.14 & 4.06 & 1.87 & 0.65 & 0.18 & 0.04 \\
\hline & $a=0.5$ & 2.55 & 5.39 & 5.52 & 3.57 & 1.65 & 0.59 & 0.17 & 0.04 \\
\hline & $a=1$ & 2.57 & 5.35 & 5.47 & 3.55 & 1.65 & 0.59 & 0.17 & 0.04 \\
\hline & $a=0.2546$ & 2.50 & 5.44 & 5.61 & 3.63 & 1.67 & 0.59 & 0.17 & 0.04 \\
\hline \multirow[t]{5}{*}{3} & $o b s$ & 1 & 6 & 4 & 1 & 1 & 2 & 1 & 0 \\
\hline & $a=0$ & 0.76 & 2.39 & 3.30 & 2.70 & 1.51 & 0.62 & 0.20 & 0.05 \\
\hline & $a=0.5$ & 0.92 & 2.32 & 2.93 & 2.40 & 1.39 & 0.60 & 0.21 & 0.06 \\
\hline & $a=1$ & 0.94 & 2.30 & 2.91 & 2.41 & 1.41 & 0.62 & 0.21 & 0.06 \\
\hline & $a=0.2546$ & 0.90 & 2.34 & 2.97 & 2.41 & 1.37 & 0.59 & 0.20 & 0.06 \\
\hline \multirow[t]{5}{*}{4} & $o b s$ & 0 & 0 & 0 & 2 & 0 & 0 & 0 & 0 \\
\hline & $a=0$ & 0.20 & 0.74 & 1.23 & 1.22 & 0.82 & 0.40 & 0.15 & 0.05 \\
\hline & $a=0.5$ & 0.25 & 0.73 & 1.11 & 1.14 & 0.85 & 0.47 & 0.20 & 0.07 \\
\hline & $a=1$ & 0.26 & 0.73 & 1.11 & 1.16 & 0.87 & 0.49 & 0.21 & 0.07 \\
\hline & $a=0.2546$ & 0.24 & 0.74 & 1.12 & 1.12 & 0.82 & 0.45 & 0.19 & 0.06 \\
\hline \multirow[t]{5}{*}{5} & $o b s$ & 0 & 0 & 1 & 0 & 0 & 0 & 0 & 0 \\
\hline & $a=0$ & 0.04 & 0.18 & 0.35 & 0.41 & 0.33 & 0.19 & 0.08 & 0.03 \\
\hline & $a=0.5$ & 0.05 & 0.18 & 0.32 & 0.41 & 0.39 & 0.29 & 0.16 & 0.07 \\
\hline & $a=1$ & 0.06 & 0.18 & 0.32 & 0.42 & 0.40 & 0.29 & 0.16 & 0.07 \\
\hline & $a=0.2546$ & 0.05 & 0.18 & 0.32 & 0.39 & 0.37 & 0.27 & 0.15 & 0.06 \\
\hline \multirow[t]{5}{*}{6} & $o b s$ & 0 & 0 & 0 & 0 & 1 & 0 & 0 & 1 \\
\hline & $a=0$ & 0.01 & 0.03 & 0.08 & 0.11 & 0.10 & 0.07 & 0.03 & 0.01 \\
\hline & $a=0.5$ & 0.01 & 0.04 & 0.08 & 0.11 & 0.14 & 0.13 & 0.10 & 0.05 \\
\hline & $a=1$ & 0.01 & 0.04 & 0.08 & 0.12 & 0.14 & 0.13 & 0.09 & 0.05 \\
\hline & $a=0.2546$ & 0.01 & 0.04 & 0.07 & 0.11 & 0.13 & 0.12 & 0.09 & 0.05 \\
\hline
\end{tabular}


We have also conducted a $\chi^{2}$ test in order to judge the goodness of fit. As usual it is important to be extremely cautious with the results of the $\chi^{2}$ test. The grouping rule we have adopted may lead to conclusions that are not matched by another grouping rules. Moreover the $\chi^{2}$ test is an asymptotic test. However we have only 79 observations in our data set.

The grouping rule we have adopted is the rule A in Lemaire (1995), i.e. all the theoretical values $>1$ and $80 \%$ of the theoretical values $>5$.

The cells have been grouped as follows: $(0,0),(0,1),(0, \geq 2),(1,0),(1,1)$, $(1,2),(1, \geq 3),(2,1),(2,2),(2,3),(2, \geq 4),(3, \geq 0),(\geq 4, \geq 0)$. The $\chi^{2}$ values as well as the associated p-values are given in the following table.

TABLE 3

GOODNESS OF FIT TEST

\begin{tabular}{lccc}
\hline & $\chi^{2}$ & $d f$ & $p$-value \\
\hline Poisson & 5.36 & 9 & 0.80 \\
PIG & 6.48 & 8 & 0.59 \\
NB & 6.42 & 8 & 0.60 \\
Hofmann & 6.42 & 7 & 0.49 \\
\hline
\end{tabular}

Based on this figures, all the fits are acceptable but the Poisson fit wins. This is coherent with the conclusions drawn after analysing the loglikelihoods.

\section{ACKNOWLEDGEMENTS}

We would like to thank both anonymous referees for their constructive remarks that led to a better presentation of the paper.

\section{REFERENCES}

AHmed, M.S. (1961) On a Locally Most Powerful Boundary Randomized Similar Test for the Independence of two Poisson Variables. Ann. Math. Statist. 32, 809-827.

Hesselager, O. (1996) Recursions for Certain Bivariate Counting Distributions and their Compound Distributions. Astin Bulletin 26, 35-52.

HofmanN, M. (1955) Uber zusammengesetzte Poisson-Prozesse und ihre Anwendungen in der Unfall versicherung. Bulletin of the Swiss Actuaries 55, 499-575.

Kestemont, R.M. and Paris, J. (1985) Sur l'Ajustement du Nombre de Sinistres. Bulletin of the Swiss Actuaries 85, 157-164.

Lemaire, J. (1995) Bonus-Malus Systems in Automobile Insurance. Kluwer Academic Publishers, Boston.

Panjer, H.H. (1981) Recursive Evaluation of a Family of Compound Distributions. Astin Bulletin 12, 22-26.

Panjer, H.H. and Willmot, G.E. (1992) Insurance Risk Models. Society of Actuaries.

Papageorgiou, H. and David, K.M. (1995) On a Class of Bivariate Compounded Poisson Distributions. Statistics \& Probability Letters 23, 93-104. 
WALhin, J.F. and PARIS, J. (2000a) Excess-of-Loss Reinsurance with Reinstatements and Ruin of the Cedent. submitted.

Walhin, J.F. and PARIS, J. (2000b) A Large Family of Discrete and Overdispersed Probability Laws. forthcoming.

JEAN-François WALHIN

SECURA Belgian Re

Rue Montoyer 12

1000 Bruxelles 